\title{
Author Index to Volume 40
}

Dentomaxillofacial Radiology (2011) 40, 537-538. doi: 10.1259/dmfr/24946707

Key to abbrevations: (BR) Book review, (CR) Case report, (C) Corrigendum, (E) Editorial, (E) Erratum, (LE) Letter to the editor, (R) Review, (RE) Research, (SC) Short communication, (SR) Systematic review, (TR) Technical report

\author{
Abe N 492 (RE) \\ Abe T 91 (RE) \\ Abou-Khalaf AE 476 (RE) \\ Akarslan ZZ 439 (RE) \\ Akgul HM 110 (RE) \\ Akinci T 160 (RE) \\ Alcaraz M 310 (SC) \\ Alcaraz-Saura M 310 (SC) \\ Al-Ekrish AA 67 (RE), 468 (LE) \\ Alhadidi A 351 (RE) \\ Allidina A 60 (CR) \\ Alsufyani NA 141 (RE), 230 (RE) \\ Anbiaee N 457 (CR) \\ Araki R 91 (RE) \\ Araujo-Pires AC 338 (RE) \\ Ariji E 133 (RE) \\ Ariji Y 133 (RE), 402 (LE) \\ Armero D 310 (SC) \\ Baker GI 60 (CR) \\ Balan A 282 (RE) \\ Banode P 213 (RE) \\ Baş O 165 (RE) \\ Becker M 315 (CR) \\ Benavente-García O 310 (SC) \\ Benson BW 328 (LE) \\ Beriat K 160 (RE) \\ Bérzin F 257 (CR) \\ Bhalla AS 403 (R) \\ Bhalla AS 147 (RE) \\ Bhowate RR 195 (CR) \\ Bhowte RR 213 (RE) \\ Bonan PR 236 (RE) \\ Bose T 282 (RE) \\ Botelho TL 257 (CR) \\ Brasileiro IV 344 (RE) \\ Bressan E 244 (RE) \\ Bruellmann DD 265 (R) \\ Brüllmann DD 170 (RE), \\ 222 (RE) \\ Bryant J 65 (LE) \\ Canteras M 310 (SC) \\ Capelozza ALA 338 (RE) \\ Cardaropoli M 506 (TR) \\ Carr A 535 (LE) \\ Castillo J 310 (SC) \\ Cevidanes LHS 351 (RE) \\ Chadwick JW 230 (RE) \\ Chandak M 213 (RE) \\ Chandak R 213 (RE) \\ Chen CSK 501 (SC) \\ Cheng Y 519 (CR) \\ Chikui T 35 (RE) \\ Christensen J 306 (SC)
}

Chu SY 451 (CR)

Coletta RD 236 (RE)

Conley R S 24 (R)

Corazza B 244 (RE)

Costa C 369 (RE)

Cotti E 506 (TR)

Courvoisier D 444 (TR)

Crozier S 184 (TR)

da Cruz Perez DE 393 (CR)

da Silveira MMF 344 (RE),

358 (RE)

Dalton J 47 (RE)

Dangore SB 195 (CR)

Daruge Júnior E 257 (CR)

de Almeida OP 393 (CR)

de Araújo NS 369 (RE)

de Carvalho FP 358 (RE)

de Moraes Ramos-Perez FM

393 (CR)

de Oliveira Santos CC 236 (RE)

de Pádua JM 393 (CR)

de Santana ST 358 (RE)

Degwekar SS 195 (CR), 213 (RE)

Dergin G 422 (RE)

d'Hoedt B 222 (RE)

Dickenson A 531 (CR)

Dölekoğlu S 379 (RE)

dos Anjos Pontual A 344 (RE)

dos Anjos Pontual ML 344 (RE), 358 (RE)

dos Santos LAN 236 (RE)

dos Santos Neto PE 236 (RE)

Dranischnikow E 265 (R)

Eickholz P 177 (RE)

Ekram M 67 (RE), 468 (LE)

El-Khateeb SM 476 (RE)

Esmaeili F 486 (RE)

Esposito S 506 (TR)

Etöz M 524 (CR)

Etöz OA 524 (CR)

Farid MM 476 (RE)

Fernandez H 310 (SC)

Fernández-Formoso N 385 (TR)

Ferreira O 338 (RE)

Fişekçioğlu E 379 (RE)

Flint DJ 328 (LE)

Flygare L 262 (LE)

Frazão MAG 358 (RE)

Fujiki T 154 (RE)

Fukunari F 415 (RE)

Fukushima Y 91 (RE)
Gadodia A 147 (RE), 403 (R)

Ganguly R 299 (RE)

Gera I 177 (RE)

Ghosh S 513 (CR)

Gomes ASL 429 (RE)

Gopal KS 191 (CR)

Goto M 133 (RE)

Gowda JS 397 (CR)

Gro $\beta$ D 265 (R)

Gupta A 251 (CR)

Gurses B 422 (RE)

Haris PS 282 (RE)

Harrell WE 24 (R)

Hashimoto K 96 (RE)

Hayashi T 126 (CR)

Healey AE 130 (CR)

Heil U 265 (R)

Hellstein J 299 (RE)

Hill ML 362 (RE)

Hirt SL 274 (RE)

Hollender LG 501 (SC)

Honda K 96 (RE)

Hsueh S 451 (CR)

Hung CF 451 (CR)

İlgüy D 379 (RE)

İlgüy M 379 (RE)

Indurkar AD 195 (CR)

Iwai K 96 (RE)

Izumi M 133 (RE)

Jabbri Khamnei H 486 (RE)

Jadu FM 60 (CR), 362 (RE)

Javadrashid R 486 (RE)

Jung N-R 434 (RE)

Junqueira JLC 369 (RE)

Kadu RP 195 (CR)

Kagawa T 415 (RE)

Kamarthi N 397 (CR)

Kamburoğlu K 439 (RE), 466 (LE)

Kang J-H 434 (RE)

Kantor ML 274 (RE)

Kapila S 24 (R)

Karadag D 160 (RE)

Karjodkar F 53 (CR), 123 (SC), 251 (CR), 471 (RE)

Karlsson L 429 (RE)

Kashima I 154 (RE)

Katsumata A 133 (RE)

Katsura K 126 (CR)

Kawagushi JT 257 (CR)
Kawasaki H 91 (RE)

Kayipmaz S 165 (RE)

Khanna V 53 (CR), 123 (SC), 251 (CR), 471 (RE)

Kiliaridis S 444 (TR)

Kim IH 274 (RE)

Kim J-D 76 (RE)

Kim J-S 76 (RE)

Kim M-S 434 (RE)

Kim S-G 76 (RE)

Kim S-H 434 (RE)

Kim Y-K 76 (RE)

Kise Y 133 (RE)

Kota S 191 (CR)

Koteeswaran D 191 (CR)

Kozai Y 154 (RE)

Krithika C 191 (CR)

Küçük M 165 (RE)

Kull L 262 (LE)

Kumar A 513 (CR)

Kumar N 463 (CR)

Kurşun Ş 439 (RE)

Kurita K 133 (RE)

Kuroiwa H 154 (RE)

Lam EWN 141 (RE), 230 (RE), 362 (RE)

Langlais RP 42 (RE), 264 (C)

Laranjeira AL 236 (RE)

Lazaridis N 84 (RE)

Lee L 60 (CR)

Lee PP 501 (SC)

Leroux BG 501 (SC)

Li B 519 (CR)

Liang H 328 (LE)

Lith A 244 (RE)

Lohe VK 195 (CR)

Long X 519 (CR)

Ludlow J 351 (RE)

Ludlow JB 115 (TR)

Lui DG 290 (RE)

Ma XC 290 (RE)

Macdonald-Jankowski DS 1 (SR)

Macleod I 535 (LE)

Maeda H 133 (RE)

Mah P 66 (LE)

Mahoney M 47 (RE), 184 (TR)

Maia AMA 429 (RE)

Mamabolo M 331 (RE)

Manohar C 513 (CR)

Margulis W 429 (RE)

Martelli-Júnior H 236 (RE)

Martínez-Beneyto Y 310 (SC) 
Martínez-Silva I 385 (TR)

Maruyama S 126 (CR)

Matzen LH 306 (SC)

Mazzoleni S 244 (RE)

McDavid WD 42 (RE), 264 (C)

Mehnert AJH 184 (TR)

Mian AZ 390 (CR)

Miki M 154 (RE)

Miloglu O 110 (RE)

Miwa K 415 (RE)

Mochizuki N 154 (RE)

Mol A 351 (RE)

Momjian A 444 (TR)

Moon Y-H 434 (RE)

Mora MJ 385 (TR)

Morita M 133 (RE)

Moroglu S 422 (RE)

Motwani MB 195 (CR), 213 (RE)

Naitoh M 133 (RE)

Nakamoto A 91 (RE)

Nakamoto N 91 (RE)

Nakazawa M 91 (RE)

Nassef MA 476 (RE)

Ng SY 102 (RE)

Nishizawa K 96 (RE)

Nityasri V 282 (RE)

Nixon PP 130 (CR)

Noffke C 331 (RE)

Noujeim M 42 (RE), 264 (C)

Öhman A 262 (LE)

Ogawa K 42 (RE), 264 (C)

Oh W-M 434 (RE)

Ohara H 91 (RE)

Okano T 42 (RE), 264 (C)

Oliveira LB 369 (RE)

Orhan K 492 (RE)

Osanai H 154 (RE)

Oz U 492 (RE)

Ozdol NC 160 (RE)
Pai KM 513 (CR)

Palladas P 84 (RE)

Pan KT 451 (CR)

Panchbhai AS 199 (R)

Panzarella FK 369 (RE)

Papachristodoulou A 84 (RE)

Park H-W 434 (RE)

Park J-Y 76 (RE)

Parrott LA 102 (RE)

Parshad R 147 (RE), 403 (R)

Patel MJ 274 (RE)

Pathak N 401 (LE)

Payne K 531 (CR)

Pedrosa RF 344 (RE)

Pharoah MJ 60 (CR)

Pilavaki M 84 (RE)

Poleti ML 338 (RE)

Prado FB 257 (CR)

Prihoda T 42 (RE), 264 (C)

Qian F 299 (RE)

Raubenheimer E 331 (RE)

Rawlani S 213 (RE)

Rilo B 385 (TR)

Röhrig B 170 (RE)

Rubira-Bullen IRF 338 (RE)

Ruprecht A 299 (RE)

Şahin B 165 (RE)

Şahman H 524 (CR)

Şekerci AE 524 (CR)

Şişman Y 524 (CR)

Saito N 390 (CR)

Sakai O 390 (CR)

Sakata Y 91 (RE)

Saku T 126 (CR)

Sansare K 53 (CR), 123 (SC), 251 (CR), 471 (RE)

Santana U 385 (TR)

Saricaoğlu ST 165 (RE)

Sato T 91 (RE)
Savage N 47 (RE)

Sawada K 96 (RE)

Schoemer E 265 (R)

Schubert MM 501 (SC)

Schulze R 170 (RE), 265 (R)

Schwanecke U 265 (R)

Scolozzi P 315 (CR), 444 (TR)

Seki K 42 (RE), 264 (C)

Seshadri S 513 (CR)

Sezgin ÖS 165 (RE)

Shams N 486 (RE)

Sharafuddeen KP 463 (CR)

Sharma R 147 (RE), 403 (R)

Sherin N 463 (CR)

Sherin S 463 (CR)

Shi H 320 (CR), 324 (CR)

Shimozato K 133 (RE)

Shiraishi T 35 (RE), 415 (RE)

Silva RF 257 (CR)

Silva-Sousa YTC 393 (CR)

Slater LJ 534 (LE)

Stellini E 244 (RE)

Styner M 351 (RE)

Su IH 451 (CR)

Sue T 42 (RE), 264 (C)

Sugino N 154 (RE)

Sugita Y 133 (RE)

Sulayman SL 170 (RE)

Suzuki M 126 (CR)

Tafakhori Z 457 (CR)

Taguchi A 154 (RE)

Takagi R 126 (CR)

Tamam C 422 (RE)

Tanaka J 91 (RE)

Terzić A 315 (CR)

Thakar A 147 (RE), 403 (R)

Thomas V 463 (CR)

Tihanyi D 177 (RE)

Timmons S 299 (RE)

Tohnak S 184 (TR)

Tomaru Y 91 (RE)

Tomasi C 244 (RE)

Torres SR 501 (SC)
Triantafyllidou K 84 (RE)

Uchida K 154 (RE)

van Rensburg LJ 400 (LE)

Venetis G 84 (RE)

Venkatraman S 397 (CR)

Vincent S 299 (RE)

Vineetha R 401 (LE)

Wada Y 91 (RE)

Wadhwa P 513 (CR)

Wang P 290 (RE), 320 (CR), 324 (CR)

Wang S 320 (CR), 324 (CR), 519 (CR)

Wenzel A 306 (SC)

Wissmeyer M 315 (CR)

Xavier CRG 338 (RE)

Xu B 320 (CR)

Yüksel S 466 (LE)

Yaffe MJ 362 (RE)

Yamada S 154 (RE)

Yamakawa T 264 (C)

Yan XB 290 (RE)

Yang S-Y 434 (RE)

Yeh CH 451 (CR)

Yeow KM 451 (CR)

Yildirim D 422 (RE)

Yildirim E 110 (RE)

Yilmaz AB 110 (RE)

Yoda T 91 (RE)

Yoo H-I 434 (RE)

Yoon S-J 434 (RE)

Yoshinari N 154 (RE)

Yoshiura K 35 (RE)

Yu Q 320 (CR), 324 (CR)

Yuasa K 35 (RE), 415 (RE)

Zhang WL 290 (RE)

Zhang Y 290 (RE) 